\title{
Mechanical rotation via optical pumping of paramagnetic impurities
}

\author{
Pablo R. Zangara, ${ }^{1}$ Alexander Wood, ${ }^{3}$ Marcus W. Doherty, ${ }^{4}$ and Carlos A. Meriles ${ }^{1,2, *}$ \\ ${ }^{1}$ Department of Physics, CUNY-City College of New York, New York, NY 10031, USA \\ ${ }^{2}$ CUNY-Graduate Center, New York, NY 10016, USA \\ ${ }^{3}$ School of Physics, The University of Melbourne, Melbourne, Victoria 3010, Australia \\ ${ }^{4}$ Laser Physics Centre, Research School of Physics, Australian National University, Canberra, \\ Australian Capital Territory 2601, Australia
}

(Received 4 April 2019; revised manuscript received 7 September 2019; published 6 December 2019)

\begin{abstract}
Hybrid quantum systems exhibiting coupled optical, spin, and mechanical degrees of freedom can serve as a platform for sensing, or as a bus to mediate interactions between qubits with disparate energy scales. These systems are also creating opportunities to test foundational ideas in quantum mechanics, including direct observations of the quantum regime in macroscopic objects. Here, we make use of angular momentum conservation to study the dynamics of a pair of paramagnetic centers featuring different spin numbers in the presence of a properly tuned external magnetic field. We examine the interplay between optical excitation, spin evolution, and mechanical motion, and theoretically show that in the presence of continuous optical illumination, interspin cross relaxation must induce rigid rotation of the host crystal. The system dynamics is robust to scattering of spin-polarized phonons, a result we build on to show this form of angular momentum transfer should be observable using state-of-the-art torsional oscillators or trapped nanoparticles.
\end{abstract}

DOI: 10.1103/PhysRevB.100.235410

\section{INTRODUCTION}

Growing applications in metrology and quantum information science are driving renewed interest in the interplay between spin and mechanical degrees of freedom, using one or the other as an interface to mediate quantized excitations [1,2]. Ingenious paths to controllably couple and manipulate spin and physical motion are also being explored as test beds for generating macroscopic quantum superposition and studying the boundaries between the quantum and classical worlds. For example, recent proposals on wave matter interferometry suggest the use of color centers in diamond as a handle to create translation [3] or rotation [4,5] superposition states in $\sim 100$-nm-size particles. Conversion between spin and mechanical rotation also lies at the heart of the Einstein-de Haas and Barnett effects [6,7], long exploited as the preferred routes to determine the effective gyromagnetic ratio of charge carriers in ferromagnetic materials. Recent extensions have built on the higher sensitivity of torsional microcantilevers to investigate, for instance, engineered magnetic multilayers [8], systems where the generation of a mechanical torque arises from domain wall displacements [9]. Other studies have examined torques generated by electron spin-flips in nanoscale systems [10], and magnetization tunneling in a single-molecule magnet coupled to a carbon nanotube resonator [11].

On a complementary front, active feedback and cavityassisted schemes have been developed to gain control of the dynamics of optically trapped dielectric particles including both their center-of-mass motion and rotation [12,13]. Driving

*cmeriles@ccny.cuny.edu this effort is the race to attain high rotation speeds as a strategy to explore centrifugal forces and vacuum-friction effects [14]. Unlike translational degrees of freedom (evolving under harmonic oscillator forces and hence characterized by equidistant energy levels), rotational degrees of freedom have a nonlinear energy spectrum and zero ground state energy, which can be exploited, e.g., to better study the superposition of rotational states in mesoscopic systems (the analog of persistent counter-propagating currents in a superconducting circuit), or for practical applications such as gyroscopy [15].

Thus far, all routes to driving particle rotation-both proposed and demonstrated-rely on the rotator's birefringence [16], or on the transfer of angular momentum from the light beam, assumed either circularly polarized or endowed of orbital angular momentum [17]. Here, we theoretically investigate an alternative form of optomechanics arising from a pair of interacting paramagnetic centers subject to light-induced spin pumping; an external magnetic field is adjusted so that the defects-assumed to have different spin numbers-can cross relax. For concreteness, we focus on the pair formed by a negatively charged nitrogen vacancy (NV) and a P1 center in diamond, though our ideas can be generalized to alternative pairs of defects, both in diamond and in other semiconductors such as $\mathrm{SiC}$. We show that in the presence of continuous optical excitation, energy-conserving spin -flips between the NV and P1 lead to a net transfer of angular momentum from the spin pair to the lattice, both in the form of spin-polarized phonons and rigid rotation of the crystal as a whole, with the latter being dominant. We find that even in the absence of external friction the system gradually slows down to attain a pseudoterminal velocity, which can be tuned by varying the applied magnetic field. With an eye on experiment, we discuss the more realistic case of particles 
(a)

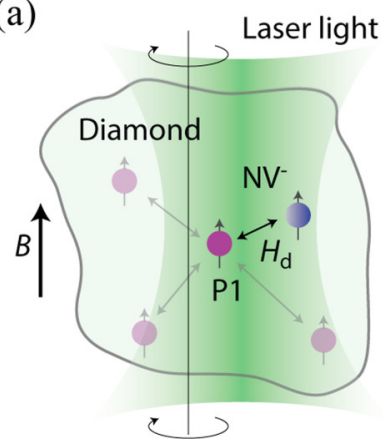

(b)

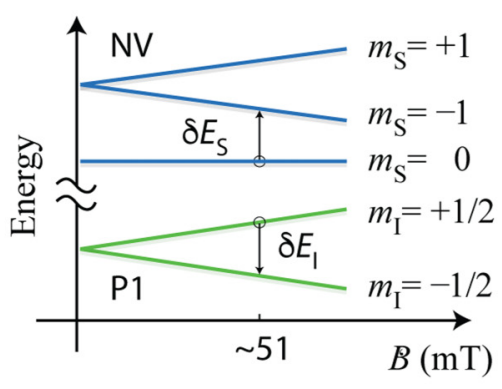

(c)

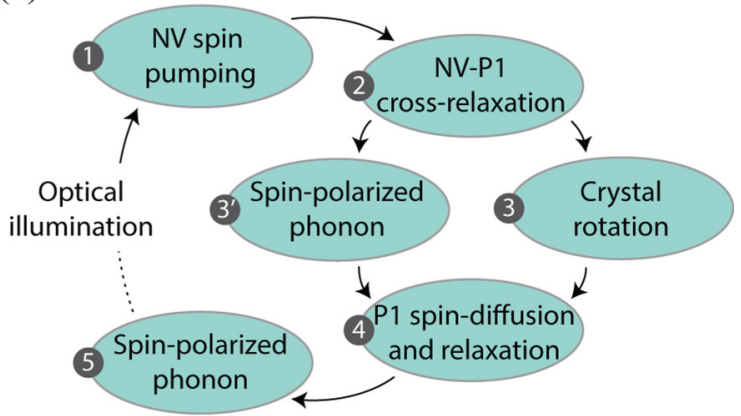

FIG. 1. The interplay between optical spin pumping and the crystal's mechanical degrees of freedom. (a) Schematics of a coupled NV-P1 pair in a diamond crystal. The P1 center interacts with other P1s farther removed from the NV. (b) Energy level diagram of the NV and P1 spins (top and bottom respectively. At $\sim 51 \mathrm{mT}$ the energies associated with each individual spin transition match, i.e., $\delta E_{\mathrm{S}} \approx \delta E_{\mathrm{I}}$. (c) Starting with NV spin optical initialization, the NV-P1 pair undergoes a cycle of cross relaxation and generation of spin-polarized phonons and rigid lattice rotation. The cycle completes with P1 spin diffusion and spin-lattice relaxation accompanied by the emission of spin-polarized phonons.

hosting multiple paramagnetic defects, and show that rigid rotation can, in principle, generate entanglement between noninteracting pairs. This finding, however, should not be seen as a practical hurdle since the coupling rate-inversely proportional to the crystal moment of inertia-is exceedingly slow, meaning that the additive action of multiple defect pairs controlled via magnetic resonance techniques can serve as a handle to act on the particle rotational dynamics.

\section{ROTATIONALLY INVARIANT HAMILTONIAN}

While the notion of spin-to-rotation conversion can, in principle, find various incarnations (see below), here we focus for concreteness on the spin pair formed by a P1 center (or neutral substitutional nitrogen impurity) and an NV center (in turn comprising a substitutional nitrogen adjacent to a vacancy). Figures 1 (a) and 1(b), respectively show a schematic and a simplified energy level diagram in the presence of an external magnetic field $B$ aligned with the symmetry axis of the NV. In its negatively charged state, the latter features a spin-1 ground state with a zero-field splitting of $2.87 \mathrm{GHz}$. Near $51 \mathrm{mT}$, the energy separation between the $m_{\mathrm{S}}=0$ and $m_{\mathrm{S}}=-1$ ground state levels of the $\mathrm{NV}$ spin matches the Zeeman splitting between the $m_{\mathrm{I}}= \pm 1 / 2$ levels of the P1. Continuous optical illumination (e.g., at $532 \mathrm{~nm}$ ) preferentially pumps the $\mathrm{NV}$ spin into the $m_{\mathrm{S}}=0$ state, from where it subsequently transitions to the $m_{\mathrm{S}}=-1$ state through dipolarfield-mediated cross relaxation with the P1. In a typical type $\mathrm{Ib}$ diamond, the P1 concentration is comparatively higher, meaning that the polarization gained by the $\mathrm{P} 1$ proximal to the source NV can easily spin-diffuse to other, farther-removed defects. The end result is a one-directional spin-pumping process, from the NV to the ensemble of P1 centers, with the P1 steady-state polarization emerging from the interplay between the P1 concentration (defining the spin diffusion constant), and the defect's spin-lattice relaxation time. This process has already been investigated using optically detected magnetic resonance both in the lab- and rotating-frames [18-21], and, more recently, has been exploited to induce high levels of ${ }^{13} \mathrm{C}$ spin polarization in diamond [22,23].

A closer inspection of the energy diagram in Fig. 1(b) indicates the above spin pumping process is deceivingly simple: While it is apparent that energy conservation can be ensured with the proper selection of the magnetic field, cross relaxation of the NV-P1 pair entails a simultaneous flip to spin states with lower quantum projection numbers, thus leading to a net reduction of the total spin angular momentum by $2 \hbar$. In other words, cross polarization of the P1 spin requires the transfer of angular momentum to "lattice" degrees of freedom, either through the generation of spin-polarized phonons [24,25], or the rigid rotation of the crystal [Fig. 1(c)].

To gain a more formal understanding, we first consider the case of a perfectly rigid solid (as we show later, a reasonable approximation for diamond). In this limit, the P1$\mathrm{NV}$ virtual-atom pair can be thought of as forming a rigid diatomic molecule, featuring the crystal's moment of inertia $\mathcal{J}$. Correspondingly, we write the system Hamiltonian as

$$
H=\Delta S_{z}^{2}+\omega_{0} S_{z}+\omega_{0} I_{z}+H_{\mathrm{d}}+\frac{L_{z}^{2}}{2 \mathcal{J}},
$$

where $\mathbf{S}(\mathbf{I})$ is the NV $(\mathrm{P} 1)$ vector spin operator, $\Delta$ is the $\mathrm{NV}$ zero field splitting, $\omega_{0} \equiv\left|\gamma_{e}\right| B$ is the electron Zeeman frequency in the magnetic field $\mathbf{B}$ (assumed along the $z$-axis and parallel to the NV), $\gamma_{e}$ denotes the electron gyromagnetic ratio, $\mathbf{L}$ is the vector operator representing the crystal angular momentum, and we assume for simplicity the system can only rotate about the $z$ axis. In Eq. (1), $H_{\mathrm{d}}$ expresses the NV-P1 dipolar interaction, here viewed as the coupling Hamiltonian $H_{\text {s-r }}$ between the spin pair and the (rigid) crystal rotation. To expose interconversion between spin and crystal rotation, we write

$$
\begin{aligned}
H_{\mathrm{s}-\mathrm{r}} \approx & H_{\mathrm{d}}=d_{0}(\mathbf{r}) \delta_{0}+d_{1}(\mathbf{r}) \lambda_{+} \delta_{1-} \\
& +d_{1}^{*}(\mathbf{r}) \lambda_{-} \delta_{1+}+d_{2}(\mathbf{r}) \lambda_{+}^{2} \delta_{2-}+d_{2}^{*}(\mathbf{r}) \lambda_{-}^{2} \delta_{2+},
\end{aligned}
$$

where $\quad \delta_{0}=S_{z} I_{z}-\frac{1}{4}\left(S_{-} I_{+}+S_{+} I_{-}\right), \quad \delta_{1 \pm}=S_{z} I_{ \pm}+S_{ \pm} I_{z}$, $\delta_{2 \pm}=S_{ \pm} I_{ \pm}$are the two-spin operators in spherical tensor form. By the same token, we denote $d_{0}=\frac{\alpha}{r^{3}}\left(1-3 \cos ^{2} \theta\right)$, $d_{1}=-\frac{3 \alpha}{2 r^{3}} \sin \theta \cos \theta e^{i \varphi}, d_{2}=-\frac{3 \alpha}{4 r^{3}} \sin ^{2} \theta e^{2 i \varphi}$, and $\lambda_{ \pm}=e^{ \pm i \phi}$. In the above expressions $\mathbf{r}=\mathbf{r}_{\mathrm{NV}}-\mathbf{r}_{\mathrm{P} 1}$ is the interspin vector with polar and azimuthal angles $\theta$ and $\phi+\varphi$, respectively; the latter is expressed as the sum of the angle $\phi$ formed by the crystal relative to the laboratory frame and the (fixed) crystal 
frame azimuthal coordinate $\varphi$. Finally, $\alpha=\mu_{0} \gamma_{e}^{2} / 4 \pi$, where $\mu_{0}$ denotes the vacuum permeability.

Assuming for now the regime where the crystal's rotational energy is smaller than the dipolar energy, we choose the external magnetic field so that $\omega_{0}=\Delta / 2$, the "energy matching" condition required for NV-P1 cross relaxation. Limiting our description to the spin subspace spanned by $\left|m_{\mathrm{S}}, m_{\mathrm{I}}\right\rangle=\{|0,+1 / 2\rangle,|-1,-1 / 2\rangle\}$, only the last two "double-flip" terms in $H_{\mathrm{d}}$ are (nearly) energy conserving, meaning that all first three contributions can be effectively truncated. In this limit, we rewrite the Hamiltonian as

$$
H=d_{2}(\mathbf{r}) \lambda_{+}^{2} \delta_{2-}+d_{2}^{*}(\mathbf{r}) \lambda_{-}^{2} \delta_{2+}+\frac{L_{z}^{2}}{2 \mathcal{J}} .
$$

Note that since $\left[L_{z}, \lambda_{ \pm}\right]= \pm \hbar \lambda_{ \pm}$, the $\lambda_{ \pm}$operators can be thought of as ladder operators to $L_{z}$. Therefore, Eq. (3) explicitly shows how angular momentum is conserved, namely, a net spin angular momentum loss from a double quantum flip is accompanied by a corresponding crystal angular momentum gain (and vice versa). The above dynamics is in strong contrast with the spin-conserving zero-quantum "flip-flops" usually governing spin diffusion processes through terms of the form $d_{0} I_{ \pm} S_{\mp}$ in $H_{\mathrm{d}}$. The key difference stems from the asymmetry created by the crystal field, acting on the NV but not the P1, and hence rendering flip-flop contributions to the Hamiltonian nonsecular.

\section{SPIN-CRYSTAL ANGULAR MOMENTUM INTER-CONVERSION}

To intuitively grasp the system dynamics in the presence of optical excitation, it is instructive to first consider the simplified case where the crystal — here seen as a free rotor-initially occupies a state $\left|m_{\mathrm{L}}\right\rangle$ of mechanical angular momentum $m_{\mathrm{L}} \hbar$, and a light pulse instantaneously projects the spin system into $|0,+1 / 2\rangle$ (we ignore for now the different initialization mechanisms in the NV and P1). Driven by the dipolar coupling, the NV-P1 pair evolves into $|-1,-1 / 2\rangle$ and, in so doing, changes the orbital part of the wave function into $\left|m_{\mathrm{L}}+2\right\rangle$. Reinitializing the spin system into $|0,+1 / 2\rangle$ repeats the process, but this time the rotor state evolves from $\left|m_{\mathrm{L}}+2\right\rangle$ into $\left|m_{\mathrm{L}}+4\right\rangle$, corresponding to buildup of the crystal angular momentum and hence to macroscopic physical rotation.

Under continuous optical excitation, this spin-induced rotational pumping can be best computed via the tight-binding representation of Fig. 2(a) where each linear chain corresponds to one of the two possible spin states and the site
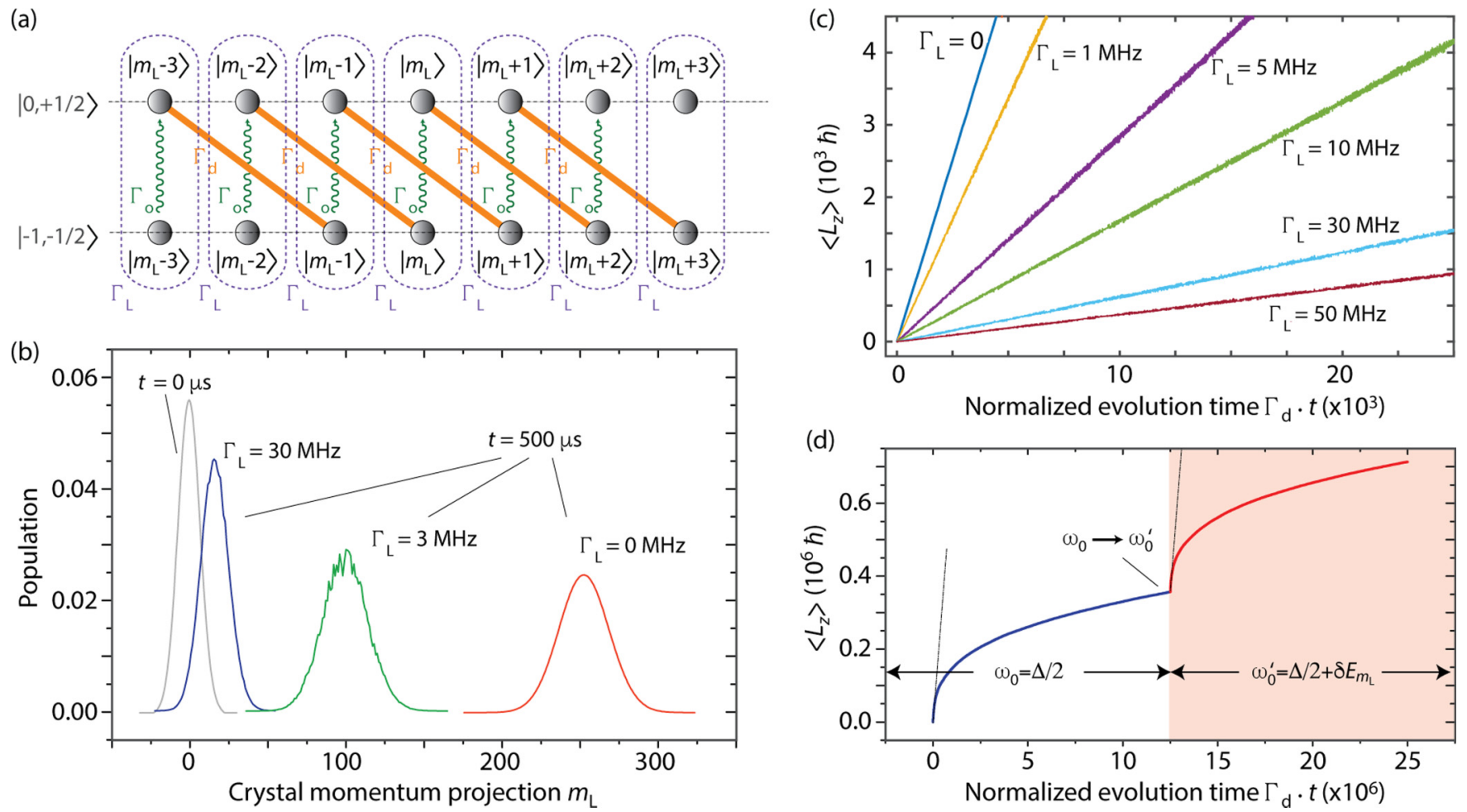

FIG. 2. Modeling spin-crystal momentum conversion. (a) "Tight-binding” representation of the rotor pumping process: Upper and lower chains correspond to spin states $|0,+1 / 2\rangle$ and $|-1,-1 / 2\rangle$, respectively, while chain sites indicate rotational states $\left|m_{\mathrm{L}}\right\rangle$. Starting from a state $\left|m_{\mathrm{S}}, m_{\mathrm{I}}, m_{\mathrm{L}}\right\rangle$, the system evolution is governed by the dipolar rate $\Gamma_{\mathrm{d}}$, the optical pumping rate $\Gamma_{\mathrm{o}}$, and the rotor decoherence rate $\Gamma_{\mathrm{L}}$. (b) Occupational probability of rotational states after evolution in the presence of continuous optical excitation for a fixed time interval $t=500 \mu \mathrm{s}$ and various rotor depahasing rates $\Gamma_{\mathrm{L}}$; the faint black trace indicates the population distribution assumed for $t=0$. The dynamics is evaluated using the Trotter-Suzuki method (see Methods); each curve shows the result after 50 averages. (c) Mean angular momentum $L_{z}$ as a function of the normalized evolution time $\Gamma_{\mathrm{d}} t$ for some rotor decoherence rates $\Gamma_{\mathrm{L}}$; the initial rotor state is that of (b). (d) Long-term evolution for the case $\Gamma_{\mathrm{L}}=0$. At sufficiently large rotational energies, the spin-crystal momentum transfer is inefficient and the system evolves towards a pseudoterminal angular speed, whose value can be adjusted by shifting the magnetic field (shaded region of the plot). In (b), (c), and (d) we use $\Gamma_{\mathrm{d}}=0.5 \mathrm{MHz}, \Gamma_{\mathrm{o}}=1 \mathrm{MHz}$, and $\hbar / 2 \mathcal{J}=10 \mathrm{~Hz}$. 
energies take values $E_{m_{\mathrm{L}}}=m_{\mathrm{L}}^{2} \hbar^{2} /(2 \mathcal{J})$. In the regime where the rotational energy is negligible (i.e., $E_{m_{\mathrm{L}}} \ll \hbar^{2}\left|d_{2}\right|$, see below), the time evolution can be cast in terms of a series of interchain hops governed by unit time probabilities $\Gamma_{\mathrm{d}}=\hbar\left|d_{2}\right| /(2 \pi)$ and $\Gamma_{\mathrm{o}}$, respectively, representing the NV$\mathrm{P} 1$ dipolar coupling and optical pumping rates. To realistically compute the system evolution, we must also take into account the rotor decoherence, which we model by imposing a dephasing rate $\Gamma_{\mathrm{L}}$ on rotation states $\left|m_{\mathrm{L}}\right\rangle$. Assuming a crystal with no net initial angular momentum (i.e., $\left\langle L_{z}\right\rangle(t=0)=0$ ) at some nonzero initial temperature $T_{\mathrm{i}}$ (i.e., $\left\langle L_{z}^{2}\right\rangle(t=0) \propto T_{\mathrm{i}}$ ), Fig. 2(b) compares the probability density of rotational states before and after a time interval $t$ of continuous optical excitation. Consistent with a crystal momentum gain, the initial distribution (faint black trace) invariably evolves to yield a net $\left\langle L_{z}\right\rangle$-as reflected by the nonzero $\left\langle m_{\mathrm{L}}\right\rangle$-with significant momentum buildup even when $\Gamma_{\mathrm{L}} \geqslant \Gamma_{\mathrm{d}}$.

Figure 2(c) shows the average angular momentum $\left\langle L_{z}\right\rangle$ as a function of time- expressed in units of the spin-crystal momentum transfer time $\Gamma_{\mathrm{d}}^{-1}$ - for various rates of dissipation $\Gamma_{\mathrm{L}}$. In all cases, we observe a linear growth, indicative of a constant torque on the crystal with value approximately proportional to $\left(\Gamma_{d}^{2}+\Gamma_{L}^{2}\right)^{-1 / 2}$. This process, however, cannot be sustained indefinitely (even if $\Gamma_{\mathrm{L}}=0$ ) since, as $\left\langle L_{z}\right\rangle$ grows, so does the crystal's rotational energy [last term in Eq. (3)], whose increasingly larger energy steps $\delta E_{m_{\mathrm{L}}} \equiv$ $E_{m_{\mathrm{L}}+2}-E_{m_{\mathrm{L}}}=2\left(m_{\mathrm{L}}+1\right) \hbar^{2} / \mathcal{J}$ become gradually comparable to the NV-P1 spin energy (i.e., $\delta E_{m_{\mathrm{L}}} \sim 2 \pi \hbar \Gamma_{\mathrm{d}}$ ), thus slowing down the spin-crystal momentum conversion [Fig. 2(d)]. Considering the extreme angular velocities demonstrated recently for optically driven nanoparticles $[12,13](\gtrsim 2 \pi \times$ $1 \mathrm{GHz}$ ), this "pseudoterminal" regime-first reached for angular frequencies of order $\sim \Gamma_{\mathrm{d}}$ - should be readily observable. Efficient rotational pumping, however, can be regained by changing the magnetic field so as to recover the "energy matching" condition, i.e., $\Delta-2 \omega_{0}^{\prime}+\delta E_{m_{\mathrm{L}}}=0$, where we use the prime to highlight the shift relative to the value $\omega_{0}=$ $\Delta / 2$ at early stages [shaded half of Fig. 2(d)].

For future reference, it is possible to use Fermi's "golden rule" to analytically calculate the rate of interconversion between NV spin polarization and crystal rotation. In the limit where $\Gamma_{\mathrm{o}} \lesssim \Gamma_{\mathrm{d}}$, we find [26]

$$
\Gamma_{\mathrm{s}-\mathrm{r}} \approx \frac{4 \pi^{2} \eta \alpha^{2}}{5 \hbar^{2} r_{\min }^{3}} \rho_{\mathrm{ss}}\left(\Gamma_{\mathrm{d}}, \Gamma_{\mathrm{L}}\right) \approx \frac{\Gamma_{\mathrm{d}}^{2}}{\left(\Gamma_{\mathrm{d}}^{2}+\Gamma_{\mathrm{L}}^{2}\right)^{1 / 2}},
$$

where $\rho_{\mathrm{ss}}\left(\Gamma_{\mathrm{d}}, \Gamma_{\mathrm{L}}\right)$ is a line-shape factor, and we assume a random distribution of NV-P1 pairs with number concentration $\eta$ and minimum separation $r_{\min }=1 \mathrm{~nm}$.

\section{INTERACTION WITH SPIN-POLARIZED PHONONS}

An alternative channel of momentum conservation is via phonons, recently shown [24,25] to carry an intrinsic "phonon-spin" angular momentum $\mathbf{L}^{\prime}=\int_{V} d^{3} r^{\prime} \rho \mathbf{u}\left(\mathbf{r}^{\prime}\right) \times$ $\dot{\mathbf{u}}\left(\mathbf{r}^{\prime}\right)$, where $\mathbf{u}\left(\mathbf{r}^{\prime}\right)$ denotes the local lattice displacement vector, $\rho$ is the crystal density, and the integral extends over the crystal volume $V$. To explicitly describe the spin-phonon interaction we express the displacement vector as $\mathbf{u}\left(\mathbf{r}^{\prime}\right)=$ $\sqrt{\hbar /(2 \rho V)} \sum_{\mathbf{k}, j} \mathbf{e}_{\mathbf{k}, j} \exp \left(i \mathbf{k} \cdot \mathbf{r}^{\prime}\right) a_{\mathbf{k}, j} / \sqrt{\omega_{\mathbf{k}, j}}+$ H.c., where
H.c. denotes Hermitian conjugate, the sum extends over all wave vectors $\mathbf{k}$ and (Cartesian) polarization branches $j, \mathbf{e}_{\mathbf{k}, j}$ denotes the phonon polarization vector, $\omega_{\mathbf{k}, j}$ is the phonon frequency, and we use the standard notation for the phonon creation and annihilation operators, respectively, $a_{\mathbf{k}, j}^{\dagger}$ and $a_{\mathbf{k}, j}$. Replacing in the expression for $\mathbf{L}^{\prime}$, one finds $[27,28]$

$$
\mathbf{L}^{\prime}=\hbar \sum_{\mathbf{k}} \frac{\mathbf{k}}{k}\left(a_{\mathbf{k}+}^{\dagger} a_{\mathbf{k}+}-a_{\mathbf{k}-}^{\dagger} a_{\mathbf{k}-}\right)
$$

where $a_{\mathbf{k} \pm}^{\dagger} \equiv \mp\left(a_{\mathbf{k} 1}^{\dagger} \pm i a_{\mathbf{k} 2}^{\dagger}\right) / \sqrt{2}$, and the positive (negative) signs indicate left (right) circular polarization. Equation (5) expresses the lattice spin angular momentum as the difference between populations of phonons, each carrying a unit $\hbar$ of angular momentum parallel or antiparallel to the direction of propagation (i.e., positive or negative quantum of angular momentum, respectively).

To include the effect of spin-polarized phonons into the model, we expand $H_{\mathrm{d}}$ to first order in the lattice displacements via the correspondence $\mathbf{r} \rightarrow \mathbf{r}+\delta \mathbf{r}$, where $\delta \mathbf{r}=\mathbf{u}\left(\mathbf{r}_{\mathrm{NV}}\right)-$ $\mathbf{u}\left(\mathbf{r}_{\mathrm{P} 1}\right)$. After some algebra, we find [26]

$$
H_{\mathrm{d}} \approx H_{\mathrm{s}-\mathrm{r}}+H_{\mathrm{s}-\mathrm{p}},
$$

where $H_{\mathrm{s}-\mathrm{r}}$ is the spin-rotation interaction derived above for the rigid rotator model [Eq. (2)], and

$$
H_{\mathrm{s}-\mathrm{p}} \approx \sum_{\mathbf{k}} i \mathbf{k} \cdot \mathbf{r}\left(b_{0} \pi_{\mathbf{k}, 0} \delta_{0}+b_{1} \pi_{\mathbf{k}, 1+} \delta_{1-}+b_{1} \pi_{\mathbf{k}, 1-} \delta_{1+}\right) .
$$

Above we use the notation $\pi_{\mathbf{k}, 0}=\left[\frac{\hbar}{2 \rho V \omega_{\mathbf{k}}}\right]\left(a_{\mathbf{k}, \mathrm{z}}-a_{\mathbf{k}, \mathrm{z}}^{\dagger}\right)$, $\pi_{\mathbf{k}, 1 \pm}= \pm\left[\frac{\hbar}{2 \rho V \omega_{\mathbf{k}}}\right]\left(a_{\mathbf{k}, \pm}^{\dagger}+a_{\mathbf{k}, \mp}\right), \quad b_{0}=-\frac{3 \alpha}{2 r^{4}} \cos \theta(1-$, $5 \cos 2 \theta)$, and $b_{1}=-\frac{3 \alpha}{16 r^{4}}(3 \cos \theta+4 \cos 3 \theta)$. We also assume that $\mathbf{r}$ is small compared to the relevant phonon wavelengths [26], i.e., $\mathbf{k} \cdot \mathbf{r} \ll 1$. As in Eq. (3), the Hamiltonian of Eq. (7) makes rotational invariance explicit, this time through the interconversion of spin and phonon angular momentum. Unlike $H_{\mathrm{s}-\mathrm{r}}$, however, $H_{\mathrm{s}-\mathrm{p}}$ connects states differing, at most, by a single quantum of angular momentum. Since individual spin flips take place at a rate $\Gamma_{\mathrm{s}-\mathrm{p}}^{(1)}$ not greater than the inverse of the spin-lattice relaxation time $T_{1}^{(\mathrm{NV})} \sim T_{1}^{(\mathrm{P} 1)}$ (induced via $H_{\mathrm{s}-\mathrm{p}}$ or other spin-lattice relaxation processes [29], typically $\sim 1 \mathrm{~ms}$ at room temperature), we conclude $\Gamma_{\mathrm{s}-\mathrm{p}}^{(1)} \ll \Gamma_{\mathrm{s}-\mathrm{r}}$.

A possibility that must be considered separately, however, is one where double spin flips are allowed via second-order processes involving simultaneous absorption and emission of phonons. In this case, the combined spin-phonon system transitions from an initial state $\left|i_{\mathbf{k}}\right\rangle=\mid \ldots n_{\mathbf{k},-}, n_{\mathbf{k},+}, \ldots, 0$, $+1 / 2\rangle$ to a final state $\left|f_{\mathbf{k}}\right\rangle=\mid \ldots n_{\mathbf{k},-}-1, n_{\mathbf{k},+}+1, \ldots$, $-1,-1 / 2\rangle$. Here, the net spin of phonons with wave vector $\mathbf{k}$-represented through spin-polarized phonon populations $n_{\mathbf{k},-}$ and $n_{\mathbf{k},+}$-grows by two units of $\hbar$, hence compensating for the angular momentum change from NV-P1 spin cross relaxation (last two quantum numbers in the kets). Note that other final states-involving, e.g., phonons with wave vector different from the initial one-are forbidden, because spin cross relaxation must conserve the total linear momentum and energy, i.e., $\mathbf{k}$ must remain unchanged.

To calculate the rate of spin-phonon momentum transfer via these second order pathways, we consider two types of 
(a)

Non-resonant phonon scattering

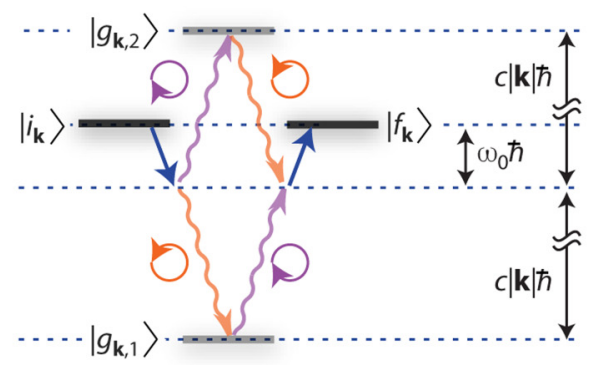

(b) Resonant phonon scattering

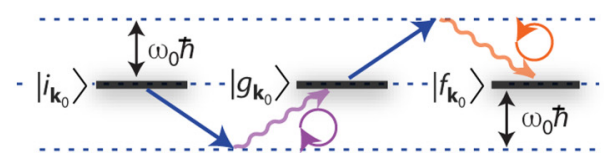

(c)

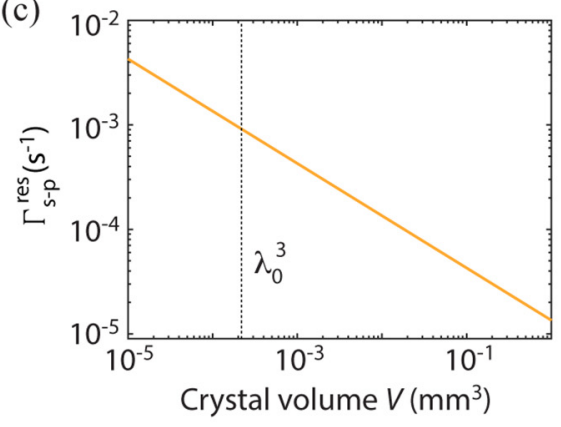

(d)

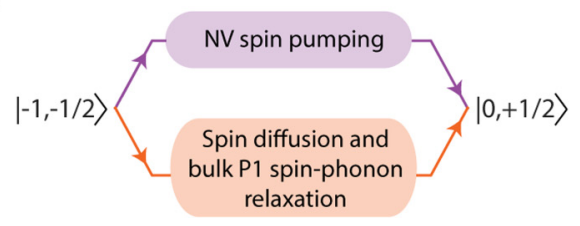

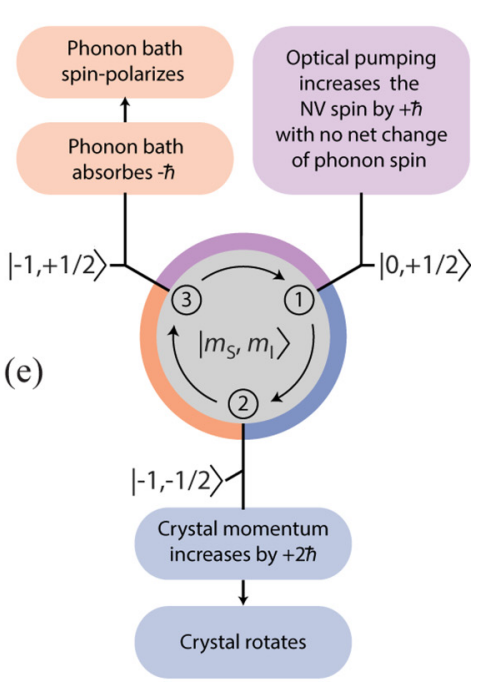

FIG. 3. Spin-phonon angular momentum conversion. (a) Nonresonant mechanism of spin-phonon conversion, i.e., $c|\mathbf{k}|>\omega_{0}$. Straight (wavy) arrows indicate spin (phonon spin) change; clockwise (counter clockwise) corresponds to phonons with negative (positive) angular momentum. The initial and final states are $\left|i_{\mathbf{k}}\right\rangle=\left|\ldots n_{\mathbf{k}-}, n_{\mathbf{k}+}, \ldots 0,+1 / 2\right\rangle$ and $\left|f_{\mathbf{k}}\right\rangle=\left|\ldots n_{\mathbf{k}-}-1, n_{\mathbf{k}+}+1, \ldots-1,-1 / 2\right\rangle$, respectively. The upper and lower virtual states are $\left|g_{\mathbf{k}, 1}\right\rangle=\left|\ldots n_{\mathbf{k}-}-1, n_{\mathbf{k}+}, \ldots-1,+1 / 2\right\rangle$, and $\left|g_{\mathbf{k}, 2}\right\rangle=\left|\ldots n_{\mathbf{k}-}, n_{\mathbf{k}+}+1, \ldots-1,+1 / 2\right\rangle$, respectively. (b) Resonant mechanism, i.e., $c\left|\mathbf{k}_{0}\right|=\omega_{0}$. The notation used for all kets is the same as in (a), except that $\mathbf{k} \rightarrow \mathbf{k}_{0}$, and the intermediate state is $\left|g_{\mathbf{k}_{0}}\right\rangle=\left|\ldots n_{\mathbf{k}_{0}-}-1, n_{\mathbf{k}_{0}+}, \ldots-1,+1 / 2\right\rangle$. (c) Resonant spin-phonon conversion rate as a function of the crystal volume; $\lambda_{0} \equiv 2 \pi c / \omega_{0}$ denotes the wavelength of resonant phonons, not supported by crystals of smaller size. (d) NV-P1 spin "reset" involving NV spin pumping and P1 spin diffusion into the bulk. Spin-phonon relaxation subsequently transfers the P1 polarization to the phonon bath. (e) Schematic representation of the NV-P1 spin cycle. Repeated sequences of NV spin pumping, P1-enabled spin cross relaxation, and NV-P1 spin resets simultaneously produce crystal rotation and polarization of the phonon bath spin.

mechanisms [26]. In the first category, we group all offresonance processes (i.e., $|\mathbf{k}|>\left|\mathbf{k}_{0}\right| \equiv \omega_{0} / c$, with $c$ denoting the speed of sound in diamond) where the transition from state $\left|i_{\mathbf{k}}\right\rangle$ to $\left|f_{\mathbf{k}}\right\rangle$ takes place via virtual states $\left|g_{\mathbf{k}, j}\right\rangle, j=1,2$ involving an NV spin flip $|0\rangle \rightarrow|-1\rangle$ and the creation (annihilation) of a phonon with positive (negative) spin [Fig. 3(a)]. The second group corresponds to resonant processes (i.e., $|\mathbf{k}|=\left|\mathbf{k}_{0}\right|$ ) involving an intermediate state $\left|g_{\mathbf{k}_{0}}\right\rangle$ with the same energy as $\left|i_{\mathbf{k}_{0}}\right\rangle$ or $\left|f_{\mathbf{k}_{0}}\right\rangle$ [Fig. 3(b)]. Despite the massive majority of nonresonant relaxation channels, this second group of processes is more efficient in inducing spin-phonon conversion of angular momentum (at least at room temperature and below), mainly because phonon states with greater wave vectors quickly depopulate due to the stiffness of diamond [26]. After a lengthy calculation, we find the characteristic spin-phonon conversion rate from these resonant, second-order processes is approximately given by the formula

$$
\Gamma_{\mathrm{s}-\mathrm{p}}^{(2)} \cong \Gamma_{\mathrm{s}-\mathrm{p}}^{(2, \text { res })} \approx \frac{\alpha}{10 c \hbar}\left(\frac{k_{\mathrm{B}} T}{\rho V}\right)^{\frac{1}{2}}\left(\frac{2 \pi \eta}{r_{\min }^{9}}\right)^{\frac{1}{4}}
$$

where $k_{\mathrm{B}}$ denotes Boltzmann's constant, and $T$ is the temperature. Interestingly, $\Gamma_{\mathrm{s}-\mathrm{p}}^{(2)}$ grows with the inverse square root of the crystal volume, implying that spin-phonon conversion is greater for diamond microparticles. Since the wavelength of resonant phonon modes $\lambda_{0} \equiv 2 \pi /\left|\mathbf{k}_{0}\right|=2 \pi c / \omega_{0}$ is of order $60 \mu \mathrm{m}$, this mechanism is quenched in sufficiently small crystals (unable to support these phonon modes). In all cases, nonetheless, we find $\Gamma_{\mathrm{s}-\mathrm{p}}^{(2)} \lesssim 10^{-1} \mathrm{~s}^{-1} \ll \Gamma_{\mathrm{s}-\mathrm{r}}$ [Fig. 3(c)], hence allowing us to conclude spin-phonon angular momen- tum conversion is not a sizable competing mechanism to spin-crystal rotation transfer.

The latter must not be interpreted, however, as implying phonons play no role in the crystal-rotation-pumping process. Phonons are key to optically repolarizing the NV center, though this process has zero net input of angular momentum into the phonon bath (see Refs. [26], [30], and [31]). On the other hand, spin-lattice relaxation of bulk P1s (which spin polarize into $|-1 / 2\rangle$ via spin diffusion from NV-coupled P1s) ultimately requires the transfer of (negative) angular momentum into the phonon bath. Therefore, the cycle of NVP1 spin initialization, evolution, and reset must be viewed as one simultaneously leading to net crystal rotation and phononbath-spin pumping, as sketched in Figs. 3(d) and 3(e).

\section{DISCUSSION AND OUTLOOK}

While our description thus far has been limited to a single NV-P1 pair, the experimental observation of spin-to-crystal momentum conversion will likely require the use of spin ensembles. Since the dynamics of each pair is coupled to the rotation of the solid-in turn, impacting all members of the ensemble-it is natural to wonder about the conditions required to treat individual contributions to the torque on the crystal as independent from each other. To address this question, we first rewrite the Hamiltonian in Eq. (3) as [26]

$$
H_{\phi}=\frac{J_{z}^{2}}{2 \mathcal{J}}+\sum_{j} d_{2, j}(\mathbf{r}) \delta_{2-, j}+d_{2, j}^{*}(\mathbf{r}) \delta_{2+, j},
$$

where $H_{\phi} \equiv U_{\phi} H U_{\phi}^{\dagger}$ with $U_{\phi} \equiv \exp \left(-i \phi\left(S_{z}+I_{z}\right) / \hbar\right)$, and $J_{z}=S_{z}+I_{z}+L_{z}$ is the total angular momentum (we denote 
$S_{z} \equiv \sum_{j} S_{z, j}, I_{z} \equiv \sum_{j} I_{z, j}$ ). The sum in Eq. (9) represents the standard (truncated) dipolar interaction of an ensemble of NV-P1 pairs in a static solid under energy matching conditions, implying that all rotation-derived effects are encapsulated in the first term. To make these effects explicit, we transform $H_{\phi}$ to the basis set where all terms in the sum are diagonal, i.e., where $U_{\mu}\left(d_{2, j}(\mathbf{r}) \delta_{2-, j}+d_{2, j}^{*}(\mathbf{r}) \delta_{2+, j}\right) U_{\mu}^{\dagger}=$ $\hbar^{2} d_{2, j}^{\prime} \mu_{z^{\prime}, j}$ with $\mu_{z^{\prime}, j}$ denoting a Pauli operator along a (pairdependent) virtual axis $z$ '. Limiting our description to the subspace involving states of crystal angular momentum $m_{\mathrm{L}}$, the Hamiltonian takes the final form

$$
\begin{aligned}
H_{\phi, \mu}^{(m)} \equiv & U_{\mu} H_{\phi}^{(m)} U_{\mu}^{\dagger} \approx-\hbar^{2} \sum_{j}\left(d_{2, j}^{\prime} \mu_{z^{\prime}, j}-(m-1) \mu_{x^{\prime}, j} / \mathcal{J}\right) \\
& +\left(\hbar^{2} / \mathcal{J}\right) \sum_{j \neq i}^{\prime}\left(\mu_{+, j} \mu_{-, i}+\mu_{-, j} \mu_{+, i}\right),
\end{aligned}
$$

where we ignore constant terms, and we assume [26] $\left\|d_{2}^{\prime}\right\| \gg$ $1 / \mathcal{J}$. In the Hamiltonian representation of Eq. (10), the first term in the upper sum can be viewed as a (local) Zeeman interaction with an effective magnetic field of amplitude proportional to the NV-P1 pair dipolar coupling, whereas the second term represents a (global) transverse field whose amplitude grows with faster crystal rotation. Finally, the primed sum (comprising only NV-P1 pairs of similar dipolar interaction strength) amounts to a rotation-induced interpair coupling term, independent of the interpair distance. Remarkably, this interaction can mediate entanglement between remote NVP1 pairs, but because the coupling amplitude is inversely proportional to the crystal's moment of inertia, long rotational coherence lifetimes - of order $\mathcal{J} / \hbar$-would be required to make this process observable. Under optical excitation, the system coherence time is dictated (at best) by the inverse optical pumping rate, $\Gamma_{\mathrm{o}}^{-1}$, much smaller than $\mathcal{J} / \hbar$ for realistic conditions. In our present regime, therefore, we can correctly describe the impact of the ensemble on the crystal dynamics simply as a sum of independent spin-pair contributions.

Experimentally observing the interplay between spin and crystalline angular momenta can capitalize on a variety of techniques explicitly conceived to sense weak forces $[32,33]$. Among them, silicon-crystal double-paddle oscillators [34]capable of detecting torques as weak as $10^{-18} \mathrm{Nm}$ at room temperature [35] — are well suited to the present application, because their large footprint can support mm-sized diamond crystals. For a crude comparison, we express the expected torque as

$$
\tau=\frac{d\left\langle L_{z}\right\rangle}{d t} \sim 2 \hbar \eta V \Gamma_{\mathrm{s}-\mathrm{r}},
$$

where we assume [26], for simplicity, near optimum NV-P1 polarization in $|0,+1 / 2\rangle$, a condition one can approach with reasonable illumination power densities of $\sim 1 \mathrm{~mW} / \mu \mathrm{m}^{2}$. For crystals with moderate NV-P1 pair concentrations $(\eta \sim$ $5 \mathrm{ppm}$ ), we find $\tau \sim 10^{-17} \mathrm{Nm}$ for optical excitation over a $50-\mu \mathrm{m}$ radius spot in a $300-\mu \mathrm{m}$-thick crystal. Further, the mechanically detected spectrum that emerges-dominated by the strong P1 hyperfine interaction with its host nitrogenserves as a signature to distinguish spin-induced torques from undesired sources $[26,36]$.
In the opposite limit of diamond nanoparticles [37-39], much higher detection sensitivities-from $10^{-21} \mathrm{Nm} / \mathrm{Hz}^{1 / 2}$ and up to $10^{-29} \mathrm{Nm} / \mathrm{Hz}^{1 / 2}$-have been predicted [40] and demonstrated [41] using optical tweezers hence making this route also feasible; in particular, sample heating (and the ensuing NV-P1 spin energy mismatch it creates [22]) can be minimized with the use of Paul traps [42-44]. As an alternative to torque sensing, here one could capitalize on schemes adapted to detecting rotational velocities via birefringenceinduced modulation of a probe laser [15]. Unlike the former, this latter strategy reveals the time integrated effect of optical excitation, and thus could help expose spin-rotation conversion in systems where the NV-P1 pair density is low.

Although our description centered on NV and P1 centers in diamond, similar derivations apply to other spin systems provided that: (i) one of the defects can be optically pumped (through spin-dependent optical excitation or via broadband illumination and spin-selective intersystem crossing); (ii) the spin numbers are different and only one has total angular momentum greater than $\frac{1}{2}$ (either in the form of an orbital singlet with spin number $S \geqslant 1$ or an orbital doublet with $S=1 / 2$ and sufficiently large spin orbit interaction); and (iii) both spins have suitably long lifetimes (so that they can be tuned in and out of resonance with sufficient change in flipflop rate that the effect can be observed). Besides the NV-P1 pair discussed herein, other defect combinations in systems such as $\mathrm{SiC}$ or garnet materials appear plausible.

Extending the ideas introduced herein promises intriguing opportunities in various uncharted fronts. For example, unlike present schemes to inducing rotation, the ability to initialize and manipulate paramagnetic centers provides a versatile handle to control the rotational dynamics of the host crystal, which could be exploited to investigate the limits of quantum superposition in mesoscopic systems. Provided the rotational coherence of the host crystal is sufficiently long, it will also be interesting to investigate the impact of rotation on the collective dynamics of the spin ensemble, which, perhaps, could lead to forms of "coherence protection" akin to that observed in heterogeneous ensembles of oscillators confined to an optical cavity $[45,46]$.

Along the same lines, the interplay between spin-lattice relaxation and chiral phonons-here found comparatively inefficient at the NV-P1 pair level-could nonetheless be exploited at the single defect level. One possibility could be to mechanically pump the NV (and/or P1) spin, for instance, by stimulating spin-polarized acoustic phonons matched to the spin resonance frequency. To this end, one could resort to existing photoacoustic methods based on timed femtosecond laser pulses [47,48], in this case tailored so as to coherently inject chiral phonons into the diamond lattice.

\section{ACKNOWLEDGMENTS}

C.A.M. thanks E. Hahn, D. Sakellariou, A.Trabesinger, and A. Pines for stimulating his interest on the topic through early discussions, and, more recently, E. Chudnovsky for fruitful conversations on the ideas herein. P.R.Z. and C.A.M. acknowledge support from the National Science Foundation (NSF) through Grants No. NSF-1619896, No. NSF-1903839, and No. NSF-1914945, and from Research Corporation for 
Science Advancement through a FRED Award; they also acknowledge access to the facilities and research infrastructure of the NSF CREST IDEALS, Grant No. NSF-HRD1547830. A.W. acknowledges support from the University of Melbourne ECR mobility fellowship scheme. M.W.D. acknowledges support from the Australian Research Council (DE170100169).

\section{APPENDIX: METHODS}

Dynamics using the Trotter-Suzuki decomposition. The tight-binding representation in Fig. 2(a) corresponds to the unitary dynamics given by the Hamiltonian in Eq. (3) and the nonunitary processes $\Gamma_{\mathrm{o}}$ and $\Gamma_{\mathrm{L}}$ (optical pumping and rotational dephasing, respectively). The standard Trotterization allows for a step-wise evolution, where the system is evolved in small time steps $\Delta t$. Here, $\Delta t$ is much smaller than the shortest time-scale in the problem, including $\Gamma_{\mathrm{d}}, \Gamma_{\mathrm{o}}$, and $\Gamma_{L}$. The projection due to optical pumping (and the reset of the P1 spin state) follows the standard quantum jump recipe [49]. In practice, this implies a stochastic projection of population from state $|-1,-1 / 2\rangle$ to the state $|0,+1 / 2\rangle$. The dephasing $\Gamma_{\mathrm{L}}$ corresponds to the analog quantum drift procedure [50], and consists in a stochastic randomization of the phase of each state $\left|m_{\mathrm{L}}\right\rangle$. The time dependence of the observable (in this case, the probability density associated to the wave function) is obtained after averaging trajectories.
[1] M. C. Kuzyk and H. Wang, Scaling Phononic Quantum Networks of Solid-State Spins with Closed Mechanical Subsystems, Phys. Rev. X 8, 041027 (2018).

[2] S. D. Bennett, N. Y. Yao, J. Otterbach, P. Zoller, P. Rabl, and M. D. Lukin, Phonon-Induced Spin-Spin Interactions in Diamond Nanostructures: Application to Spin Squeezing, Phys. Rev. Lett. 110, 156402 (2013).

[3] C. Wan, M. Scala, G. W. Morley, ATM. A. Rahman, H. Ulbricht, J. Bateman, P. F. Barker, S. Bose, and M. S. Kim, Free Nano-Object Ramsey Interferometry for Large Quantum Superpositions, Phys. Rev. Lett. 117, 143003 (2016).

[4] Y. Ma, T. M. Hoang, M. Gong, T. Li, and Z.-Q Yin, Proposal for quantum many-body simulation and torsional matter-wave interferometry with a levitated nanodiamond, Phys. Rev. A 96, 023827 (2017).

[5] T. Delord, L. Nicolas, Y. Chassagneux, and G. Hétet, Strong coupling between a single nitrogen-vacancy spin and the rotational mode of diamonds levitating in an ion trap, Phys. Rev. A 96, 063810 (2017).

[6] G. G. Scott, Review of gyromagnetic ratio experiments, Rev. Mod. Phys. 34, 102 (1962).

[7] J. E. Losby, V. T. K. Sauer, and M. R. Freeman, Recent advances in mechanical torque studies of small-scale magnetism, J. Phys. D 51, 483001 (2018).

[8] T. M. Wallis, J. Moreland, and P. Kabos, Einstein-de Haas effect in a NiFe film deposited on a microcantilever, Appl. Phys. Lett. 89, 122502 (2006).

[9] S-H. Lim, A. Imtiaz, T. M. Wallis, S. Russek, P. Kabos, L. Cai, and E. M. Chudnovsky, Magneto-mechanical investigation of spin dynamics in magnetic multilayers, Europhys. Lett. 105, 37009 (2014).

[10] G. Zolfagharkani, A. Gaidarzhy, P. Degiovanni, S. kettemann, P. Fulde, and P. Mohanty, Nanomechanical detection of an itinerant electron spin flip, Nat. Nanotechnol. 3720 (2008).

[11] M. Ganzhorn, S. Kyatskaya, M. Ruben, and W. Wernsdorfer, Quantum Einstein-de Haas effect, Nat. Commun. 711443 (2016).

[12] R. Reimann, M. Doderer, E. Hebestreit, R. Diehl, M. Frimmer, D. Windey, F. Tebbenjohanns, and L. Novotny, GHz Rotation of an Optically Trapped Nanoparticle in Vacuum, Phys. Rev. Lett. 121, 033602 (2018).

[13] J. Ahn, Z. Xu, J. Bang, Y.-H. Deng, T. M. Hoang, Q. Han, R.-M. Ma, and T. Li, Optically Levitated Nanodumbbell
Torsion Balance and GHz Nanomechanical Rotor, Phys. Rev. Lett. 121, 033603 (2018).

[14] A. Manjavacas, F. J. Rodríguez-Fortuño, F. J. Garciíade Abajo, and A. V. Zayats, Lateral Casimir Force on a Rotating Particle near a Planar Surface, Phys. Rev. Lett. 118, 133605 (2017).

[15] Y. Arita, M. Mazilu, and K. Dholakia, Laser-induced rotation and cooling of a trapped microgyroscope in vacuum, Nat. Commun. 4, 2374 (2013).

[16] K. D. Wulff, D. G. Cole, and R. L. Clark, Controlled rotation of birefringent particles in an optical trap, Appl. Opt. 47, 6428 (2008).

[17] H. Shi and M. Bhattacharya, Optomechanics based on angular momentum exchange between light and matter, J. Phys. B 49153001 (2016).

[18] S. Armstrong, L. J. Rogers, R. L. McMurtrie, and N. B.Manson, $\mathrm{NV}-\mathrm{NV}$ electron-electron spin and $\mathrm{NV}-\mathrm{N}_{S}$ electron-electron and electron-nuclear spin interaction in diamond, Phys. Proc. 3, 1569 (2010).

[19] J. D. A. Wood, D. A. Broadway, L. T. Hall, A. Stacey, D. A. Simpson, J. P. Tetienne, and L. C. L. Hollenberg, Wide-band nanoscale magnetic resonance spectroscopy using quantum relaxation of a single spin in diamond, Phys. Rev. B 94, 155402 (2016).

[20] A. Laraoui and C. A. Meriles, Approach to dark spin cooling in a diamond nanocrystal, ACS Nano 7, 3403 (2013).

[21] C. Belthangady, N. Bar-Gill, L. M. Pham, K. Arai, D. Le Sage, P. Cappellaro, and R. L. Walsworth, Dressed-State Resonant Coupling Between Bright and Dark Spins in Diamond, Phys. Rev. Lett. 110, 157601 (2013).

[22] D. Pagliero, K. R. Koteswara Rao, P. R. Zangara, S. Dhomkar, H. H. Wong, A. Abril, N. Aslam, A. Parker, J. King, C. E. Avalos, A. Ajoy, J. Wrachtrup, A. Pines, and C. A. Meriles, Multispin-assisted optical pumping of bulk ${ }^{13}$ Cnuclear spin polarization in diamond, Phys. Rev. B 97, 024422 (2018).

[23] R. Wunderlich, J. Kohlrautz, B. Abel, J. Haase, and J. Meijer, Optically induced cross relaxation via nitrogen-related defects for bulk diamond ${ }^{13} \mathrm{C}$ hyperpolarization, Phys. Rev. B 96, 220407(R) (2017).

[24] L. Zhang and Q. Niu, Angular Momentum of Phonons and the Einstein-de Haas Effect, Phys. Rev. Lett. 112, 085503 (2014).

[25] D. A. Garanin and E. M. Chudnovsky, Angular momentum in spin-phonon processes, Phys. Rev. B 92, 024421 (2015). 
[26] See Supplemental Material at http://link.aps.org/supplemental/ 10.1103/PhysRevB.100.235410 for detailed accounts on (i) the derivation of spin-to-crystal conversion rate formulas, (ii) a formal description of interaction between cross relaxation and spin-polarized phonons, (iii) angular momentum conservation (light, spin, phonon) in the NV spin optical pumping cycle, (iv) a derivation of the rotation-driven interspin-pair interaction formula, and (v) potential experimental demonstrations.

[27] E. Merzbacher, Quantum Mechanics (Wiley, New York, 1961).

[28] J. D. Jackson, Classical electrodynamics, $3^{\text {rd }}$ ed. (Wiley, New York, 1991).

[29] A. Abragam and B. Bleaney, Electron Paramagnetic Resonance of Transition Ion (Clarendon Press, Oxford, 1970).

[30] K.-M. C. Fu, C. Santori, P. E. Barclay, L. J. Rogers, N. B. Manson, and R. G. Beausoleil, Observation of the Dynamic Jahn-Teller Effect in the Excited States of NitrogenVacancy Centers in Diamond, Phys. Rev. Lett. 103, 256404 (2009).

[31] M. L. Goldman, A. Sipahigil, M. W. Doherty, N. Y. Yao, S. D. Bennett, M. Markham, D. J. Twitchen, N. B. Manson, A. Kubanek, and M. D. Lukin, Phononinduced Population Dynamics and Intersystem Crossing in Nitrogen-Vacancy Centers, Phys. Rev. Lett. 114, 145502 (2015).

[32] H. B. Chan, V. A. Aksyuk, R. N. Kleiman, D. J. Bishop, and F. Capasso, Nonlinear Micromechanical Casimir Oscillator, Phys. Rev. Lett. 87, 211801 (2001).

[33] B. Ilic, H. G. Craighead, S. Krylov, W. Senaratne, C. Ober, and P. Neuzil, Attogram detection using nanoelectromechanical oscillators, J. Appl. Phys. 95, 3694 (2004).

[34] T. H. Metcalf and X. Liu, An ultra-high Q silicon compound cantilever resonator for Young's modulus measurements, Rev. Sci. Instrum. 84, 075001 (2013).

[35] L. Haiberger, M. Weingran, and S. Schiller, Highly sensitive silicon crystal torque sensor operating at the thermal noise limit, Rev. Sci. Instrum. 78, 025101 (2007).

[36] T. Wolf, P. Neumann, K. Nakamura, H. Sumiya, T. Ohshima, J. Isoya, and J. Wrachtrup, Subpicotesla Diamond Magnetometry, Phys. Rev. X 5, 041001 (2015).

[37] V. R. Horowitz, B. J. Alemán, D. J. Christle, A. N. Cleland, and D. D. Awschalom, Electron spin resonance of nitrogen-vacancy centers in optically trapped nanodiamonds, Proc. Natl. Acad. Sci. USA 109, 13493 (2012).
[38] M. Geiselmann, M. L. Juan, J. Renger, J. M. Say, L. J. Brown, F. J. Garcia de Abajo, F. Koppens, and R. Quidant, Threedimensional optical manipulation of a single electron spin, Nat. Nanotechnol. 8, 175 (2013).

[39] T. M. Hoang, J. Ahn, J. Bang, and T. Li, Electron spin control of optically levitated nanodiamonds in vacuum, Nat. Commun. 7, 12250 (2016).

[40] T. M. Hoang, Y. Ma, J. Ahn, J. Bang, F. Robicheaux, Z.-Q. Yin, and T. Li, Torsional Optomechanics of a Levitated Nonspherical Nanoparticle, Phys. Rev. Lett. 117, 123604 (2016).

[41] S. Kuhn, B. A. Stickler, A. Kosloff, F. Patolsky, K. Hornberger, M. Arndt, and J. Millen, Optically driven ultra-stable nanomechanical rotor, Nat. Commun. 8, 1670 (2017).

[42] T. Delord, P. Huillery, L. Schwab, L. Nicolas, L. Lecordier, and G. Hetet, Ramsey Interferences and Spin Echoes from Electron Spins Inside a Levitating Macroscopic Particle, Phys. Rev. Lett. 121, 053602 (2018).

[43] T. Delord, L. Nicolas, L. Schwab, and G. Hétet, Electron spin resonance from $\mathrm{NV}$ centers in diamonds levitating in an ion trap, New J. Phys 19, 033031 (2017).

[44] G. P. Conangla, A. W. Schell, R. A. Rica, and R. Quidant, Motion control and optical interrogation of a levitating single nitrogen vacancy in vacuum, Nano Lett. 18, 3956 (2018).

[45] T. Zhong, J. M. Kindem, J. Rochman, and A. Faraon, Interfacing broadband photonic qubits to on-chip cavity-protected rare-earth ensembles, Nat. Commun. 8, 14107 (2017).

[46] Z. Kurucz, J. H. Wesenberg, and K. Mølmer, Spectroscopic properties of inhomogeneously broadened spin ensembles in a cavity, Phys. Rev. A 83, 053852 (2011).

[47] O. Synnergren, T. N. Hansen, S. Canton, H. Enquist, P. Sondhauss, A. Srivastava, and J. Larsson, Coherent phonon control, Appl. Phys. Lett. 90, 171929 (2007).

[48] D. H. Feng, X. Q. Pan, X. Li, T. Q. Jia, and Z. R. Sun, Coherent acoustic phonon generation and detection by femtosecond laser pulses in ZnTe single crystals, J. Appl. Phys. 114, 093513 (2013).

[49] M. B. Plenio and P. L. Knight, The quantum-jump approach to dissipative dynamics in quantum optics, Rev. Mod. Phys. 70, 101 (1998).

[50] L. J. Fernández-Alcázar and H. M. Pastawski, Decoherent timedependent transport beyond the Landauer-Büttiker formulation: A quantum-drift alternative to quantum jumps, Phys. Rev. A 91, 022117 (2015). 